\title{
SOUNDSCAPE CLOCK: SOUNDSCAPE COMPOSITIONS THAT DISPLAY THE TIME OF DAY
}

\author{
Abdullah Ismailogullari \\ University of Hamburg \\ Institute for Systematic Musicology \\ Neue Rabenstraße 13 \\ 20354 Hamburg, Germany \\ a.ismail@mailbox.org
}

\author{
Tim Ziemer \\ University of Hamburg \\ Institute for Systematic Musicology \\ Neue Rabenstraße 13 \\ 20354 Hamburg, Germany \\ tim.ziemer@uni-hamburg.de
}

\begin{abstract}
This paper presents an ambient auditory display that communicates the time of day. Four soundscapes represent different quadrants of the clock. Auditory icons divide the quadrants into three parts that represent hours, and four partitions that represent every quarter of an hour. The auditory display is little intrusive and only informative to those who are privy to its principles. Suitable application areas are offices where staff can derive the time from the soundscape, while customers stay unaware and may only enjoy the calm, auditory nature scene. To experience the calm ambient character of the auditory display we suggest you to play the demo while reading the paper: https://tinyurl.com/y4yd8zkh.
\end{abstract}

\section{INTRODUCTION}

Receiving the natural soundscape in urban areas and especially inside buildings can be complicated and sometimes even impossible. Natural soundscapes deliver information about what is happening around us. They consist of daily and seasonal rhythms and patterns $[1$, pp. $76-77]$. These patterns provide information like about the time of day, for instance the singing of a rooster in the morning or the seasonal appearance of various animals and their distinct sound. We suggest to generate soundscape compositions that can deliver information like natural soundscapes do.

Mostly, people are used to audible alarms as auditory displays, like the ringing of a phone, that grab our attention immediately. In contrast to that, ambient auditory displays [2] are non-intrusive and should not distract the listener. Hence, careful sound design is necessary to be informative, but at the same time pleasant, unintrusive and not attention-capturing, annoying or even stressful. In this paper, we present an ambient auditory display, that combines soundscape compositions and auditory icons [3] for peripheral awareness of daytime. Staff privy to the auditory display principles can derive the rough time of the day from the sound scene, and extract more precise time information when consciously paying attention. At the same time, the auditory display is non-intrusive. Customers stay uninformed and may enjoy the nature soundscape, while employees are not distracted by the sounds.

(c) (1) (8) This work is licensed under Creative Commons Attribution Non Commercial 4.0 International License. The full terms of the License are available at http://creativecommons.org/licenses/by-nc/4.0

\section{BACKGROUND AND RELATED WORK}

Studies suggest that the impact of nature sound improves the recovery after stress compared to noise [4]. Nature sounds have been shown to mask annoying background sounds in offices [5]. Hui Ma and Shan Shu found that soundscapes had a stronger impact on psychological restoration in simulated open-plan offices compared with visual scenes and both continuous and intermittent had positive effects [6]. Birdsongs have been found to influence perceived naturalness, annoyance, and pleasantness of road traffic noise environments with low sound levels [7]. Fountain sounds sometimes reduced perceived loudness of traffic noise and birdsongs significantly enhanced soundscape pleasantness and eventfulness [8]. Water sound have been found to enhance urban soundscapes the most [9] and the pleasantness of water fountains in public spaces was positively correlated with their temporal variability [10]. So besides perceiving nature sounds as pleasant [11], they offer an opportunity to protect from a less pleasant and potentially distracting effect of environmental noise [12].

Many auditory display studies are concerned with intrusiveness. Fredrik Kilander and Peter Lönnqwist present a concept that uses audio cues to deliver information like incoming mail [13], [14]. The concept is meant for personal and shared space. For the election of sound cues, they attach much importance to intrusiveness of sound. They propose sounds that are easily recognisable and natural to minimise intrusion. Ralf Jung and Tim Schwartz use a location based approach to deliver personalized information [15]. Their concept bases on background music, which is meant to stay peripheral and avoid too much attention. Within the music they embed audio cues consisting of user specific instruments. These are rhythmically and melodically adjusted to the background music, and directed to the position of the user, by playing it through the closest speaker. Hanna Zoon, Saskia Bakker and Berry Eggen present with Chronoroom Clock [16] a study on sonification of time. Their design provides the time of day based on the location of a sound source. It consists of many small piezo speakers on the wall, that are placed in a circle. Each plays a sound for a certain time, then the sound is played by the next speaker. Its design is similar to a usual wall clock, which makes it easy to interpret.

\section{SOUNDSCAPE DESIGN}

People tend to check the time of the day frequently to stay aware. Sometimes it is needed immediately and it is critical for the next 
activity. Sometimes it is less urgent but still important. This need leads to checking out the time on devices with visual displays. In some situation this behavior can be seen rude by other people, for example when being at a meeting. Even in everyday situations, the possibility of being aware of the time without taking out the mobile phone or turning the body from the area of attention appears attractive. By making the time audible the information can be sensed without moving.

\subsection{Sonification of Time}

A 12-hour clock represents the time of the day. This 12-hour period is divided into four three-hour quadrants. Each quadrant is subdivided into the concrete hour. Hours are divided into quarters. This hierarchy reflects the degree of consciousness necessary to interpret the detail of the daytime: Peripheral awareness is necessary to interpret quadrants of the day. Conscious counting is necessary to interpret the exact hour. However, counting from one to three should still allow task sharing, so interpreting the hour should not distract the staff too much from other tasks. Additional focus to the timing of events is necessary to interpret the quarters of an hour. At this detail level deriving the time may be as distracting as looking at a watch. Nonetheless, concentrating on sound may seem less interruptive and impolite to the customer than looking at a watch.

\subsubsection{Quadrants of the day}

Four Scenes represent quadrants of the day. Scenes are different compositions of natural soundscapes. Example scenes are illustrated in Fig. 1, these are Seashore, Rustling Leaves, River and Bonfire. We made the order of the scenes appropriate to time of the day. It starts with a calm and gentle Seashore Soundscape. Rustling Leaves is more restless. River then is more calm but steady. The last scene is made for late evening/night. The Bonfire scene is appropriate, as it suggests comfort and a homely feeling. This scene also contains the sound of a cricket to imply nighttime. The sound compositions contribute to the comfort of the customer and for the staff the scenes serve to mask noise and reduce stress, while delivering a rough estimate of the daytime that needs little attention to interpret.

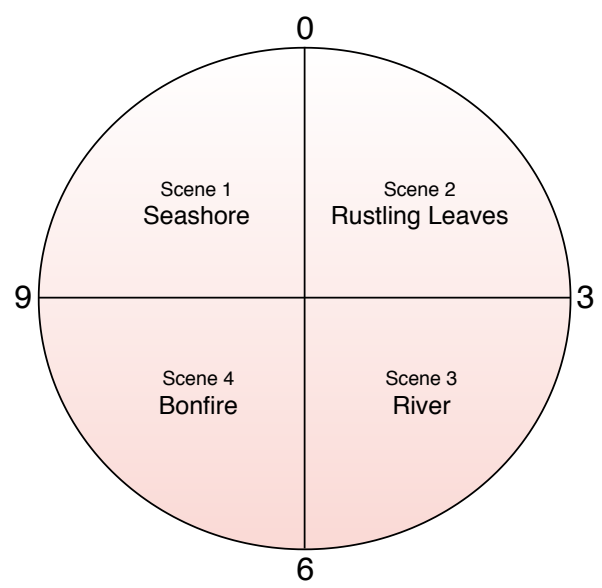

Figure 1: Example of scenes that consists of four different soundscape compositions.

\subsubsection{Hours}

Hours are represented by a bell sound as an auditory icon. The sound represents the hour within the quadrant, i.e., the clock strikes one to three times. Assuming the example in Figure 1 one bell strike represents $9,12,3$ or 6 o'clock, two bell strikes $10,1,4$ or 7 o'clock and three bell strikes $11,2,5$ or 8 o'clock.

\subsubsection{Quarters of an hour}

In a way, the constellation of bell strike and birdsong can be considered as an earcon: The temporal distance of birdsong to the bell sound indicates quarters of an hour. Figure 2 illustrates the four temporal settings at which the birdsong is played. The bell icon in Figure 2 represents the temporal position of the bell sound. A birdsong that starts shortly before the bell sound means "quarter to half past". A birdsong shortly after means "half past to quarter to". The other two settings are temporally more distant to the bell sound. The one that is played before the bell sound indicates "sharp to quarter past". Whereas the other one indicates "quarter to sharp". The temporal distance of a birdsong to a bell sound can be 1 to 5 seconds.

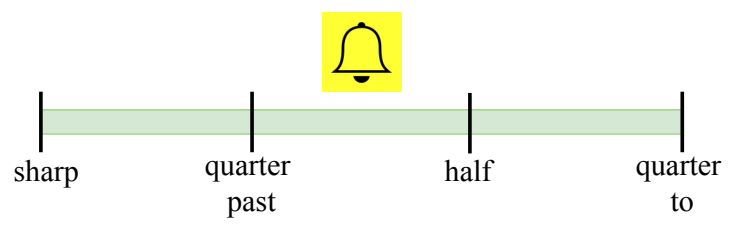

Figure 2: Temporal settings at which the birdsong is played.

\subsection{Implementation}

As part of this study, four scenes were created with Pure Data [18]. Those compromise scenes with rustling leaves, seashore, small river and bonfire. A possibility for the creation is to record natural soundscapes und playback the sampled sound. We decided to use Granular Synthesis [19] and also generate sound according to Procedural Audio [20]. With Granular Synthesis sampled sound can be manipulated and new sound is created by resynthesis. By Procedural Audio we mean the creation of sound models, like a wind-model to create wind sounds, that are controlled by physically meaningful parameters, like speed of wind.

We developed software that provides many parameters to design the compositions and the mapping of information on the fly. We also do not need hours of recordings to avoid repetitiveness. Because these scenes are designated to be played over weeks or months, we think the risk of repetitiveness may become an issue. With Granular Synthesis we only need recordings up to a minute length. By random variation of typical granulation parameters [19] like playback rate (Pitch and tempo shift), amplitude, grain length and sample position, we get an output that is enormously diverse. Alongside that we developed models of wind, fire and animals that are based or inspired by examples from Andy Farnell [21, pp. $327-649]$. These models provide many possibilities to modify the sound. By randomizing the parameters we get a constantly evolving and changing output. The bell sound is created by adding sines with different Amplitude-Envelopes (Additive Synthesis). The birdsong is created with Frequency Modulation Synthesis. The parameters of the Frequency Modulation and the shape 
of the Amplitude-Envelope are randomized, so that every birdsong has a unique character.

\subsection{Example}

A demo of the soundscape clock is available at https:// tinyurl.com/y $4 \mathrm{yd} 8 \mathrm{zkh}$. The demo is 12 minutes long and contains three minutes of each of the soundscape compositions that are illustrated in Fig. 1. For the purpose of demonstrating the variations of the bell strike-birdsong patterns, we showcase different times for every minute. The demo starts with the seashore scene at 9 o'clock sharp. The other times that are displayed are listed in the description to the demo. The scenes are not repetitive except for the $0.1 \mathrm{~Hz}$ amplitude modulation of the wind model. This model is used in the second and third scene of the demo. Its spectrogram is plotted in Fig. 3. The spectral distribution is held between brown and pink noise, which can mask high-frequency sound and is more pleasing than noise with more high-frequency energy [22], [23].

\section{DISCUSSION}

In this paper, we presented a concept for an ambient auditory display. Like other studies [13], [14], [15], [16], our design is highly concerned with intrusiveness of sound. We use soundscape compositions as a framework within which information is placed and displayed to the user. It is intended to run in the background. Information that is placed inside is supposed to be well integrated, so that it becomes a part of the composition. Such a design can offer information in a way that Weiser and Brown introduce as calm technology [24]. "Calm technology engages both the center and the periphery of our attention, and in fact moves back and forth between the two" [24, p. 81].

Our concern is to provide an overall awareness of time without creating too much attention and disturbance. This goal is achieved by embedding auditory icons as part of a soundscape composition. A design choice for the icons could be the use of sounds that are plausible in the scene, like a typical animal sound to be found in a natural soundscape. The icon for displaying the minutes, the birdsong, is such a choice. Then again, noticeability of the sounds seems also important to consider. For example when birdsong is already a component of the scene, adding another as an auditory icon can be confusing. Bell sound on the other hand is a very recognizable sound, so there is a risk that it grabs too much attention. Besides the choice of the sounds, we think that psychoacoustic metrics [25] like sharpness, roughness, loudness and tonality are important to consider for these goals. In our case we smoothen the amplitude rising at the beginning of the bell sound to reduce the sharpness of the attack.

We chose to display the auditory icons once every minute. The challenge is to be not too disturbing by a high recurrence rate but still be around when a user needs to know the time. A frequent appearance of the icons can blend out other parts of the composition. When it becomes too foregrounded, it can potentially ruin the composition, because it is not being perceived as an authentic soundscape. Once a minute is possibly already too short. Our choice is made up on the reflection of the situation that a user needs to know the time and waits for the icons to appear. By using a period of more minutes, the waiting could quickly become annoying.

We want to keep the display of time easy, so that it is mentally not challenging. By using the sequence of scenes to already deliver the quadrants, the information that is delivered by our auditory icon for hours is simplified. The number of bell sounds that has to be counted is restricted to a maximum of three. Counting needs particular attention, which is counterproductive for our goals. The displaying of the minutes is also kept easy to reduce consciousness necessary to interpret. As a result our display of minutes is very rough.

By using soundscape compositions we show an opportunity for auditory displays that can also enhance the auditory field of rooms like workspaces. We think that the idea to cover information by using natural soundscape composition is a valuable contribution, because it keeps our auditory icons less intrusive. Additionally, soundscapes are known for having positive effects and there is a requirement to generate them for places such as workspaces.

\section{CONCLUSION AND FUTURE WORK}

This paper presented a concept of how to use soundscape compositions as auditory displays. We use it to deliver the time of day. Other information that is workplace related, or personal, can be incorporated in the composition. For example mail income or, like mentioned by Kilander et al., the coworker presence, by assigning everyone to a certain birdsong could be displayed [13]. Delivering personal information at places that are used for public could be difficult because in our concept information is not directed to a chosen person. The use of our concept should depend on the character of the information. If the information is a case of emergency, using birdsong in a natural soundscape composition may be less suitable.

To deliver quadrants we use different soundscape compositions. The order of these is in our case intended to be appropriate to the time of day. The election and order of these could also depend on the season, the room and location they are played in or the taste of the user. Besides, we think that scenes do not have to be specific to certain real places. Because people are used to the blending with human made sounds, we also think the choice of sound for auditory icons can be diverse without seeming inappropriate.

We mentioned that natural soundscapes consists of daily and seasonal rhythms and patterns. A prospective research could be a close examination of these pattern. We could gather details on how natural soundscapes provide information, possibly about the time, season and weather, and how these affect humans. In this manner, we could generate soundscapes that work equivalent to the real ones. From this examination, we could also gather a better understanding that helps to design appropriate sonifications to provide various information. For such an attempt, our implementation in Pure Data is very suitable, because every component is synthesized or processed, and therefore offers parameter that potentially enables each to provide information. Possibly the whole generated soundscape can provide information.

For future work the concept as shown has to be evaluated by users. Our use of different compositions in a sequence and the design of the auditive clock have to be tested. In a basic evaluation case that tests the readability of the clock, subjects would first be taught about the meaning of the components. Afterwards the compositions would be played to them whereby they have to write down the time that is provided by it in succession. Besides that, it is also important to evaluate the intrusiveness of our concept in a real life case study, for example at a workplace. To examine whether the auditory display is in fact ambient to those who are not privy to the sound principles, and informative to those who are, a 


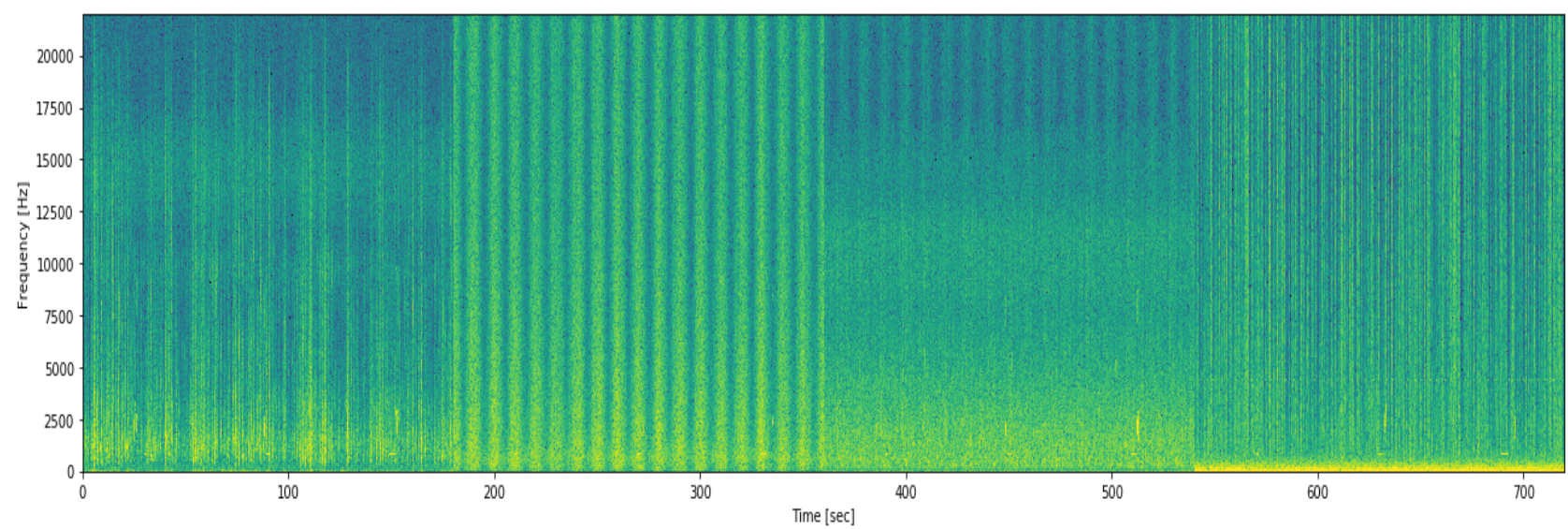

Figure 3: Spectrogram of a 12-minute soundscape clock demo including four different scenes.

test installation and questionnaire as in [26] could be employed. In this way, many choices we made, such as the components or the period of time, could be analyzed.

\section{REFERENCES}

[1] B. Truax, Acoustic Communication. Noorwood, New Jersey: Ablex Publishing Corporation, 1984.

[2] S. Ferguson, "Sonifying every day: Activating everyday interactions for ambient sonification systems," in Proceedings of the 2013 International Conference on Auditory Display (ICAD 2013), Lodz, Poland, July 6-10 2013.

[3] W. W. Gaver, "Auditory icons: Using sound in computer interfaces," Hum.-Comput. Interact., vol. 2, no. 2, pp. 167-177, June 1986.

[4] J. J. Alvarsson, S. Wiens, and M. E. Nilsson, "Stress recovery during exposure to nature sound and environmental noise." International Journal of Environmental Research and Public Health, vol. 7, no. 3, pp. 1036-1046, March 2010.

[5] A. G. DeLoach, J. P. Carter, and J. Braasch, "Tuning the cognitive environment: Sound masking with "natural" sounds in open-plan offices," The Journal of the Acoustical Society of America, vol. 137, no. 4, p. 2291, 2015.

[6] H. Ma and S. Shu, "An experimental study: The restorative effect of soundscape elements in a simulated open-plan office," Acta Acustica united with Acustica, vol. 104, no. 1, pp. 106-115, 2018.

[7] Y. Hao, J. Kang, and H. Wrtche, "Assessment of the masking effects of birdsong on the road traffic noise environment," The Journal of the Acoustical Society of America, vol. 140, no. 2, pp. 978-987, 2016.

[8] B. D. Coensel, S. Vanwetswinkel, and D. Botteldooren, "Effects of natural sounds on the perception of road traffic noise," The Journal of the Acoustical Society of America, vol. 129, no. 4, pp. EL148-EL153, 2011.

[9] J. Y. Jeon, P. J. Lee, J. You, and J. Kang, "Perceptual assessment of quality of urban soundscapes with combined noise sources and water sounds," The Journal of the Acoustical Society of America, vol. 127, no. 3, pp. 1357-1366, 2010.
[10] M. Rdsten Ekman, P. Lundn, and M. E. Nilsson, "Similarity and pleasantness assessments of water-fountain sounds recorded in urban public spaces," The Journal of the Acoustical Society of America, vol. 138, no. 5, pp. 3043-3052, 2015.

[11] K.-C. Lam, L. Brown, L. Marafa, and K.-C. Chau, "Human preference for countryside soundscapes," Acta Acustica united with Acustica, vol. 96, no. 3, pp. 463-471, May 2010.

[12] J. Errett, E. Eileen Bowden, M. Choiniere, L. M Wang, E. Ryherd, E. , and W. , "Effects of noise on productivity: Does performance decrease over time?" in AEI 2006: Building Integration Solutions - Proceedings of the 2006 Architectural Engineering National Conference, vol. 1. American Society of Civil Engineers (ASCE), March 2006, pp. 221228.

[13] F. Kilander and P. Lönnqvist, "A weakly intrusive ambient soundscape for intuitive state perception," in Continuity in Future Computing Systems, J. Doherty, M. Massink, and M. Wilson, Eds. Oxford: The Central Laboratory of the Research Councils, 2001, pp. 70-74.

[14] _ "A whisper in the woods - an ambient soundscape for peripheral awareness of remote processes," in Proceedings of the 2002 International Conference on Auditory Display (ICAD 2002), Kyoto, Japan, July 2-5 2002.

[15] R. Jung and T. Schwartz, "Peripheral notification with customized embedded audio cues," in Proceedings of the 13th International Conference on Auditory Display (ICAD 2007), Montreal, Canada, June 26-29 2007.

[16] H. Zoon, S. Bakker, and J. Eggen, "Chronoroom clock : peripheral time awareness through sound localization," in Proceedings of the 17th Annual Conference on Auditory Display (ICAD 2011), Budapest, Hungary, June 20-24 2011.

[17] M. Puckette, "Pure data," in International Computer Music Conference, San Francisco, USA, 1996, pp. 224-227.

[18] J. P. Leonard, "Granulation of sound in video games," in AES 41st International Conference, London, UK, February 2-4 2011.

[19] A. Farnell, "Procedural audio theory and practice," in The Oxford Handbook of Interactive Audio, K. Collins, B. Kapra- 
los, and H. Tessler, Eds. New York: Oxford University Press, 2014, pp. 531-540.

[20] - Designing Sound. London, England: The MIT Press, 2010.

[21] M. Gardner, Fractal music, hypercards and more. New York, United States: W.H. Freeman and Company, 1992.

[22] T. Ziemer, H. Schultheis, D. Black, and R. Kikinis, "Psychoacoustical interactive sonification for short range navigation," Acta Acustica united with Acustica, vol. 104(6), pp. 1075-1093, 112018.

[23] M. Weiser and J. S. Brown, "The coming age of calm technology," in Beyond Calculation: The Next Fifty Years of Computing. New York, NY: Springer New York, 1997, pp. $75-85$.

[24] H. Fastl and E. Zwicker, Psychoacoustics. Facts and Models, 3rd ed. Berlin, Heidelberg: Springer-Verlag, 2007.

[25] E. Brazil and M. Fernstrm, "Investigating ambient auditory information systems," in Proceedings of the 13th International Conference on Auditory Display (ICAD 2007), Montreal, Canada, June 26-29 2007. 